\title{
Dynamic Performance Modeling and Analysis of B2 - K1016 CNC camshaft grinder bed
}

\author{
Jinwei Fan ${ }^{1,}$, Lanqing Zhang ${ }^{2, \mathrm{~b}}$ and Hongliang Wang ${ }^{2, \mathrm{c}}$ \\ ${ }^{1}$ School of Mechanical Engineering, Beijing University of Technology, Beijing 100124, China; \\ ${ }^{2}$ School of Mechanical Engineering, Beijing University of Technology, Beijing 100124, China. \\ ajwfan@bjut.edu.cn, b1002878319@qq.com, ${ }^{\mathrm{c}}$ 977714868@qq.com
}

Keywords: CNC camshaft grinder; Pro/e; ANSYS; finite element analysis; optimal design;

\begin{abstract}
It is a measure of the dynamic performance of the machine tool design merits of the important performance indicators.This paper use Pro/E and ANSYS Workbench integrated development platform, for a certain type of grinder bed for three-dimensional modeling and modal analysis to improve dynamic performance, and based on the results of the various parts of the structure size and internal ribs rational layout optimization and design, so as to provide a theoretical basis for the camshaft grinding machine design.
\end{abstract}

\section{Introduction}

B2 - K1016 CNC camshaft grinder is the Beijing Machine Tool Works CO., LTD., to design, to develop high-end CNC machine tool for camshaft grinding.CNC camshaft grinding machine as the work of the camshaft machining machine tool, needs to have a reasonable structure and dynamic performance, and in its various components, the bed is an extremely important structural bulky. Size design and layout form of the bed structure, plays a decisive role in its stiffness and modal properties of the merits. Due to deficiencies of the bed dynamic stiffness and structure of the bed design unreasonable, which often led to its generating substantial vibration in working condition, cause greater relative amplitude occurs between the workpiece and the cutting tool, thus reducing the grinding machine precision. Therefore, the dynamic characteristics of the bed and its size, shape and layout of the bed ribs have a close relationship. This paper uses finite element analysis software, through three-dimensional modeling of the grinder bed and finite element analysis, and the comparative analysis of a series of calculated results, get the optimal design scheme, so as to guide the design of the camshaft grinding machine lathe bed.

\section{Organization of the Text}

\section{CNC camshaft grinder bed Finite Element Modeling}

Three-dimensional modeling of the grinder bed. Because grinder bed itself complicated structure, first carries on the reasonable simplified, using Pro/E for hree-dimensional modeling. B2 K1016 CNC camshaft grinding bed is T the layout of the whole casting structure and before lathe bed height and after the bed height is equal to $620 \mathrm{~mm}$.Before bed height is $650 \mathrm{~mm}$; the rear bed height is $550 \mathrm{~mm}$; the before bed length has $2280 \mathrm{~mm}$, width $400 \mathrm{~mm}$, the bed length $800 \mathrm{~mm}$, width $650 \mathrm{~mm}$. Before bed rails have V- flat, bed casting together with. The projection plane of the after bed is used to install the plate and the projection plane formed by two rectangular ribs support. Around the bed has a corresponding surface water to reflux coolant.Internal bed body arranged five transverse ribs.The intermediate rib has for wiring and take the tube cavity; bed thickness and the thickness of the ribs are $30 \mathrm{~mm}$. Behind, side and bottom of the bed have the windows, a lot of the hole at the bottom, used to reduce the weight of the bed.

Bed solid model is established by Pro/E drawing the appropriate sketch to stretch and cut.Characteristics by having the same structure of the ribs can be used to quickly generate an array manner to establish the basic physical structure. Using Pro/ E to built three-dimensional model bed as shown in Figure 1) 


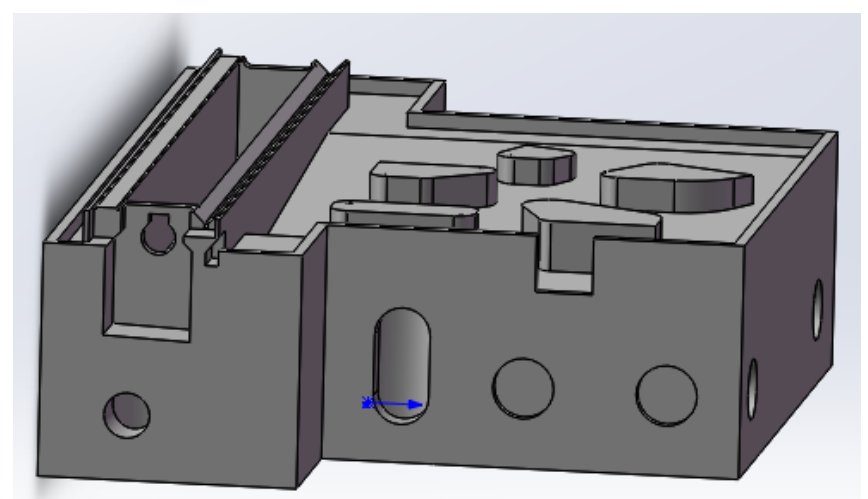

Fig. 1 Three-dimensional model of the body

Using ANSYS analysis of the solid model. Define material properties. The bed is made of gray cast iron HT200, unit corresponding elastic modulus $\mathrm{E}=1.2 \mathrm{e} 11 \mathrm{~Pa}$, Poisson's ratio $\mu=0.25$, density $\rho=7200 \mathrm{~kg} / \mathrm{m}^{3}$.

Mesh.The central task of finite element modeling is the discrete structure, namely the mesh. In ANSYS meshing can be freely mapped meshing, drag and swept meshing and mixed meshing. Generally, the finer the mesh, the higher the accuracy, but it also increases calculation time. As this bed model is more complex, manual meshing and mapping of the grid is more diffficult.we use automatic meshing, medium size, divided in bed cell size control in 50 to meet the accuracy requirements. Bed finite element mesh shown in Fig 2)

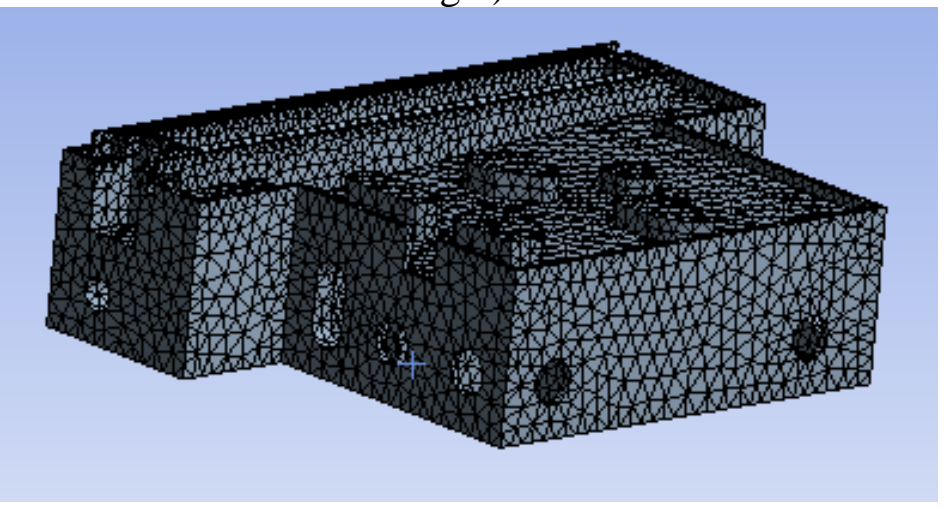

Fig. 2 Bed finite element mesh model

Constraints. CNC camshaft grinder bed is installed on the pad iron, so the pad iron bottom is imposed face constraints, limiting the y-direction of the corresponding node degrees of freedom and the z-direction and body vertical rotational degree of freedom.

Results of modal analysis. Through the modal analysis calculating the natural frequency and vibration mode of the structure could be made during the design and improvement of structural natural frequency to avoid in the process of using the external excitation frequency.Grinder Bed is a continuum.Quality and flexibility are continuously distributed, with an infinite number of modal, but the low order modal large effect on the dynamic characteristics of vibration system.By analyzing the extracted bed six natural frequencies and formation as shown in Table 1)

Table 1 Body original model six natural frequencies and mode shapes

\begin{tabular}{c|c|c}
\hline Order Number & Inherent Frequency $(\mathrm{HZ})$ & \multicolumn{1}{c}{ Mode Shapes } \\
\hline 1 & 590.53 & Before the bed around Y axis \\
\hline 2 & 613.59 & The overall reverse \\
\hline 3 & 689.65 & Before the bed wall bending \\
\hline 4 & 702.13 & Before the bed wall bending \\
\hline 5 & 767.36 & Before the bed wall bending \\
\hline 6 & 791.75 & Guide torsion plus local bending \\
\hline
\end{tabular}



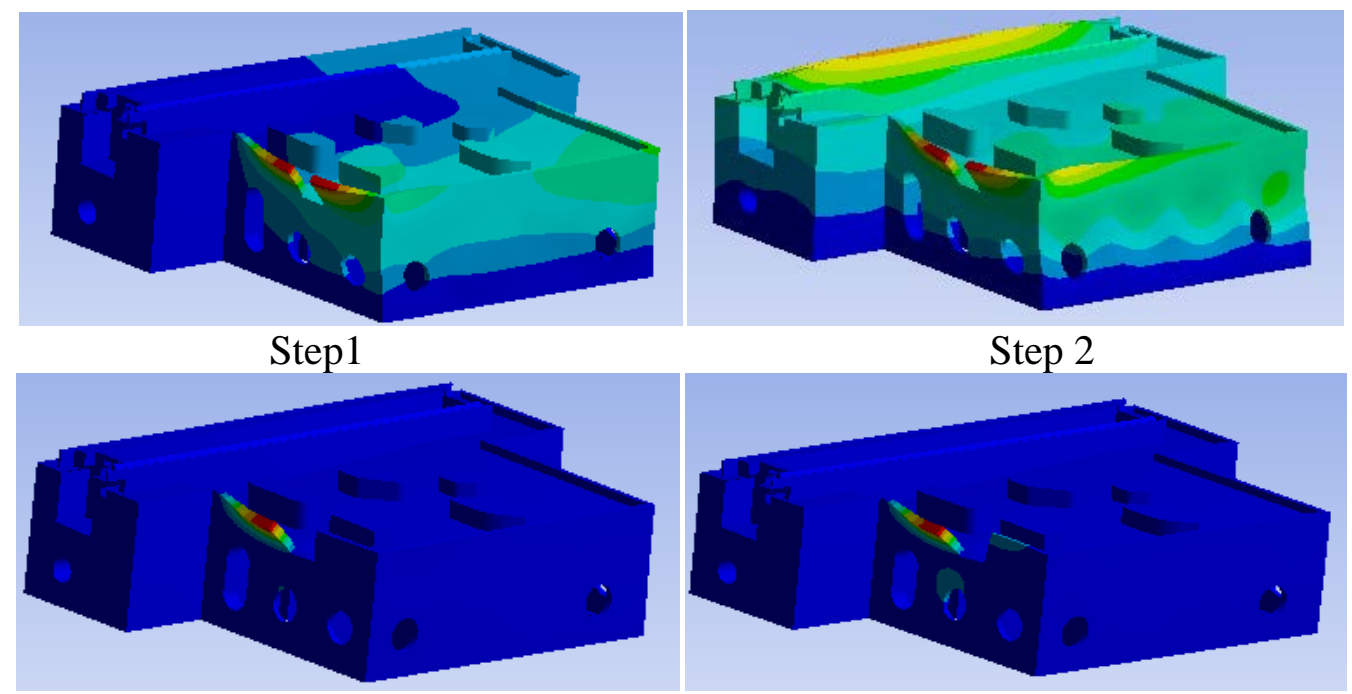

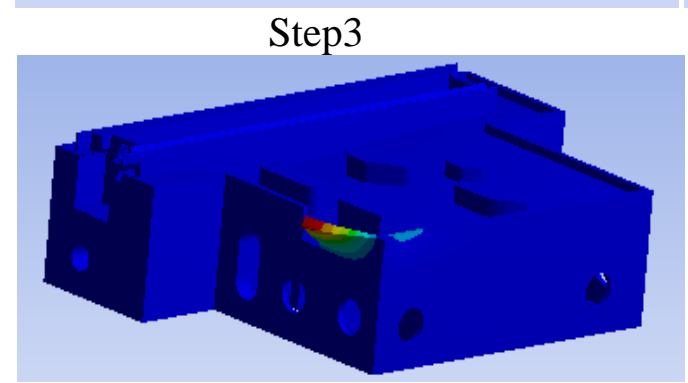

Step 5

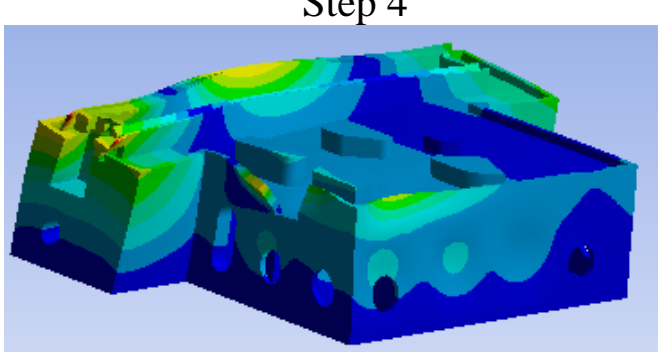

Step 6

Fig. 3 Bed before six order modal deformation maps

Optimized design of the bed. By the ANSYS analysis results, the bed before two order natural frequency is low, close to the main shaft of the excitation frequency, easy to produce resonance phenomenon which has a great influence on the torsion deflection of lathe bed, thus affecting the workpiece machining accuracy.From the bed deformation amount of displacement, former central lathe bed and the side of the before bed open the window, thus optimizing its focus on design.

In this paper, by improving bed structure size and the internal layout of the two-stage front ribs to increase the natural frequency, thereby reducing the amount of deformation of the bed to improve machining precision of grinding machines.

\section{Design of bed structure.}

Grinder bed on both sides of the window itself is intended to reduce the weight of the bed, but also easy to clean sand during casting. Therefore, to meet the conditions may be improved shape of the window, such as a triangle or circle. It may be appropriate to increase the wall thickness of the sides of the front bed.

Changing rib thickness. Considering the grinder operating band can be considered that the first few modal characteristics of the bed has an important role. Now whichever frequency to improve before the second-order basis, we made the following improvements.

Increasing the wall thickness, rib thickness.Considering the wall thickness and steel plate thickness in an increase will increase the quality of lathe bed, the bed overweight brings inconvenience when casting lathe bed and will produce crack, affecting the processing quality. In order to explore the lathe bed thickness impact on the natural frequency, we will increase the thickness of controlled within $5 \mathrm{~mm}$.

Improved a1 type: ribs thickness increases $2 \mathrm{~mm}$.

Improved a2 type: bed wall thickness increases $2 \mathrm{~mm}$.

Improved a3 type: ribs and bed wall thickness increase $2 \mathrm{~mm}$.

Improved a4 type: rib thickness increases $4 \mathrm{~mm}$.

Improved a5 type: bed wall thickness increases $4 \mathrm{~mm}$.

Improved a6 type: rib and bed wall thickness increase $4 \mathrm{~mm}$.

Layout and design of ribs. The former central lathe bed can be optimized mainly by way of 
laying out the rib plates and can be designed as a "well", "W", and "X" types.

It can be seen from the analysis, in the above several improved scheme, especially in the changing number of stiffened plate and the shape which is most evident influence on the natural frequency.The second is the hole size and shape of the hole.the last is to increase the wall thickness and steel plate thickness that have a minimum effect on natural frequencies. The actual improvements can consider the impact of these factors on the bed stiffness, designed bed structure in Figure 4, which can greatly enhance the natural frequency of the original bed to enhance its rigidity.

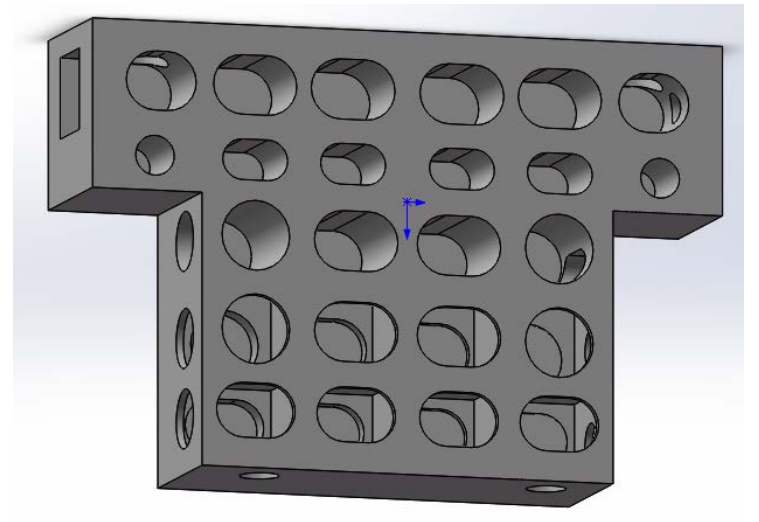

Fig. 4 improved lathe bed three-dimensional model diagram

\section{Summary}

Solving the problem of engineering technology by using the method of finite element analysis is indispensable role in engineering design .In the grinder bed of structural design , ANSYS analysis software enables designers to effectively solve design problems. In this paper, the B2 - K1016 CNC camshaft grinder bed has carried on the modal analysis, by study of low-order natural frequencies and mode shapes, obtaining bed deformation characteristics, thus getting the weaknesses of the bed's tructural rigidity. Then a variety of design schemes are compared, which concluded that optimal design scheme, laiding the foundation for optimal design of bed.

\section{References}

[1] Tlusty J, Ismail F. Dynamic Structural IdentificationTask and Method[J]. Annals of the CIRP, 1980,29: 238-247.

[2] Lee J M. A Study on the Dynamic Modeling of Structure with Bolted and Bearing Joints[J]. Annals of the CIRP, 1988,37: 136-144.

[3] Hull, Partick V. Optional synthesis of compliant mechanisms using subdivision and commercial FEA[J]. American Society of Mechanical Engineers 2006,25: 83-86.

[4] J.M. Vance, T.P Yeh. Computer Aided Dsign Improvement of Machine tools[J]. Structure Incoporting Joint Dynamics Date,1979,128(1):391-396.

[5] T. jiang, M Ciredast. Virtual assembly using virtual reality techniques[J]. Computer-Aided Design, 1997,29(8):575-584.

[6] Wang Zhiming, Yang jianguo, Wang Guoqing, Zhang Genbao, Reliability evaluation of numerical control machine tools using Weibull mixture models[J]. Advanced Materials Research, Advanced Materias Science and Technology, 2011(181-182): 161-165.

[7] Yang Zhaojun, Hao Qingbo, chen Fei, Xu Binbin, Li Xiaobing,Zhao Hongwei, A comprehensive fuzzy reliability allocation method of NC machine tools based on interval analysis[J]. Beijing Gongye Xuebao/Journal of Harbin Institute of Technolege, 2011, 43(3): 321-329. 